\title{
Kleine Notizen über parasitische Pilze Japans.
}

Von

Seiya Ito.

Bei den Arbeiten über die Krankheiten unserer Pflanzen kommen dann und wann Pilze zur Beobachtung, die teils neu, teils unvollständig bekannt sind. Der Bearbeitung derartiger Formen sind die folgenden Zeilen gewidmet.

\section{Sclerospora Sacchari T. Miyake.}

Im Jahre 1910 fand T. Mryake auf dem Versuchsfelde der Regierungsversuchsstation für die Zuckerindustrie in Daimokko (Formosa) eine gefährliche Blattkrankheit des Zuckerrohrs (Saccharum officinarum L.), die sich später ebenfalls in fast allen Distrikten nachweisen liess. Der äusserst schädliche Pilz, der diese Krankheit verursacht, erwies sich als eine neue Art der Gattung Sclerospora, die Sclerospora Sacchari T. Mryake n. sp. benannt wurde. Mryakes schöne Arbeit über diese Krankheit erschien in japanischer Sprache im: Report of Work of the Sugar Experiment Station, Government of Formosa, Bull. 1, 1911, S. 1-61, mit 9 Tafeln und 4 Karten. Die Diagnose dieses Pilzes lautet wie folgt:-

Maculis striatis, primum flavis, demum rufo-brunneis efformantibus; mycelis inter cellulas crescentibus, non septatis; haustoriis vesiculæformibus vel irregularibus; cæspitulis inconspicuis, albidis; hyphis conidiophoris pauce (1-2 in numero) erumpentibus, $160-170 \mu$ longis, basi $10-15 \mu$ crassis ; ramis 2 vel 3 partitis, rarius septatis, hyalinis; conidiis plerumque 
ellipsoideis, apice rotundatis, basi leniter papillatis vel rotundatis, $25-41 \times 15-23 \mu$, rarius $49-54 \times 19-23 \mu$, hyalinis, granulosis, germ-tubo germinantibus ; oogoniis ellipsoideis, rufobrunneis, $55-73 \times 49-58 \mu$; oosporis sphæroideis, $40-50 \mu$ in diam., episporio crasso $(3.8-5.0 \mu)$, intense flavo.

$\mathrm{Hab}$. in foliis vivis Sacchari officinaris in Formosa (Japonia) crescentis.

Es ist eine sehr interessante Tatsache, dass die Konidien von Sclerospora Sacchari wie bei Peronospora vermittelst eines Keimschlauches, der aus einer beliebigen Stelle der Seitenwand hervordringt, keimen. Gestützt auf die Resultate schlage ich an dieser Stelle vor, die Gattung Sclerospora in 2 -Untergattungen zu trennen, gemäss der Differenz des Keimungsprozesses.

1. Subgenus :-Eusclerospora.

Conidia zoosporas gignentia.

2. Subgenus :-Peronosclerospora.

Conidia directe germinantia.

Neuerdings hat G. S. KuLKarni auf den Blättern von Andropogon Sorghum in Indien eine neue Varietät von Sclerospora graminicola entdeckt, die var. Andropogonis Sorghi n. var. benannt und in den Memoirs of the Dep't of Agric. in India, Botanical Series, Vol. V, No. 5, 1913, S. 268-274, mit 2 Tafeln publiziert wurde. Die Konidien dieses Pilzes keimen auch mit einem Keimschlauch. Der Pilz ist der zweite Vertreter der Untergattung Peronosclerospora.

$\mathrm{Ob}$ der indische Pilz mit Sclerospora Sacchari identisch ist, wird sich definitiv erst nach Vergleichungs- und Kulturversuchen entscheiden lássen.

\section{Ustilago Rottbœlliæ Syd. et Butr.}

In den Ann. Mycol. Bd. V (1907) S. 486 haben H. et P. Sydow und E. J. Butrer den Brandpilz, den Butler auf den Rispen von Rottbœllia compressa L. in Pusa (Indien) beobachtet hatte, unter diesem Namen beschrieben, und danach hat SACCARDo die Beschreibung aus obiger Zeitschrift in seiner Sylloge Fungorum XXI, S. 499 abgedruckt. 
Neuerdings hat I. Mryake die Ustilago Rottbolliz Miyake n. sp., die er in China (Iichang, Hupei, Sept. 1908) gesammelt hatte, in dieser Zeitschrift (Vol. XXVII, No. 314, Tokyo, 1913, S. 42) beschrieben und abgebildet. Der Autor hat dabei folgende Bemerkung angefügt: „, Auf Rottbœllia-Arten habe ich keine Ustilago-Art gefunden, daher hielt ich den Pilz für eine neue Species."

Das Auftreten von Ustilago auf Rottbœllia in Japan ist noch nicht mitgeteilt worden. Auf meiner botanischen Exkursion im Sommer 1910 in der Provinz Echigo fand ich brandige Rispen von Rottbœllia compressa unten am Ninoji-Berge bei Shibata. Die Eigenschaften des Pilzes sind folgende :-

Diese Art verwandelt den ganzen Blïtenstand in eine sehr stäubende, schwarze oder bräunlich-schwarze Sporenmasse, die anfangs von grauer Epidermis umschlossen ist. Bisweilen entwickelt sich die Sporenmasse nur in den Fruchtknoten. In beiden Fällen bleibt häufig beim Verfliegen der Sporen nur die schmale Achse zurück. Die Sporen sind hellbraun, kugelig oder ellipsoidisch, betragen $6-8 \mu$ in Durchmesser, mit diinner (zirka $0.5 \mu)$, glatter Membran.

Ausserdem habe ich die Specimen dieses Pilzes, die K. Yoshino im August 1900 in Santo-Gun, Provinz Echigo, gesammelt hatte, und die mir Herr. Prof. Dr. K. Miyabe freundlichst zur Verfügung stellte, untersucht.

Dass alle indischen, chinesischen und japanischen Pilze zu einer Art gehören, folgt aus den Beschrcibungen von Sydow, Butler und Miyake; meine eigenen Beobachtungen stimmen in den wichtigsten Punkten mit denen dieser Autoren völlig überein. Diese Art muss daher jetzt den Namen Ustilago Rottbœlliæ Syd. et Butl. führen.

Puccinia Epimedii (Henn. et Shrr.) Miyabe et Ito.

Aecidiis = Aecidium Epimedii Henn. et ShIr. in Englers Bot. Jahrb., XXVIII, 1901, (264).

Soris teleutosporiferis hypophyllis, maculis orbicularibus vel suborbicularibus, $3-4 \mathrm{~mm}$ diam. brunneis insidentibus, medio- 
cribus, 1-2 mm diam., sparsis, rotundatis, epidermide semper tectis, compactis, atris ; teleutosporis clavatis vel oblongis, apice rotundatis, truncatis vel leniter acutiusculis, parum incrassatis $(2-7 \mu)$, medio plerumque leniter constrictis, basi attenuatis, levibus, dilute flavo-brunneolis, 34-62 × 10-19 $\mu$; pedicellis brevibus brunneis; mesosporis in formis variabilibus, 25-33 $\times 9-13 \mu$; paraphysibus brunneis, numerosis, inter se collectis, septam sporas tribubus separatam formantibus.

Hab. in foliis vivis Epimedii macranthi in Japonia crescentis.

Prov. Echigo, in monte Yakushi (Julio 1905)-Yahagi (Julio 22, 1908)-in monte Yahiko (Julio 23, 1908 ; Aug. 1912).

P. Hennings hat eine neue Aecidium-Art, die M. Shirai auf den Blättern von Epimedium macranthum Morr. et DCNE. in der Izu-Provinz gesammelt hatte, unter dem Namen Aecidium Epimedii Hens. et SHIr. in Englers botanischem Jahrbuch (1901) beschrieben und dabei folgende Bemerkung beigefügt:„, Eine äusserst zierliche Art, deren cylindrische, etwas gekrümmte Pseudoperidien locker in gelblichen Flecken stehen." Diese Aecidien sind sehr weit in unserem Gebiete verbreitet. Es fehlte bis jetzt eine Mitteilung uiber ihre Teleutostadien.

Im Juli 1905 beobachtete ich zum erstenmal auf EpimediumBlättern auf dem Berg Yakushi in der Echigo-Provinz ein schwarzes, kompaktes Teleutosporenlager auf einem Blattflecken, der mit solchen, die von Aecidium verursacht werden, grosse Aehnlichkeit besass. Später sammelte ich die neue Puccinia-Art an verschiedenen Orten in dieser Provinz. Leider unternahm ich keine Impfungsversuche zur Darstellung des genetischen Zusammenhangs der Aecidien und Teleutosporen. Doch konnte ich häufig sehen, dass diese beiden Formen gemeinsam auf einem Blatte wuchsen. Aus diesem Grunde führe ich diese Pilzfornen vorläufig unter dem obigen Namen als eine Art auf.

\section{Gymnosporangium japonicum Syd.}

Im Jahre 1899 hat zuerst P. Sydow den auf dem Stengel 
von Juniperus chinensis L. lebenden Pilz, welchen M. ShIraI in Tokyo (Komaba) gefunden hatte, in Hedwigia Bd. XXXVIII, Beiblatt S. 141 beschrieben. Im nächsten Jahre wies M. SHIRAI den Zusammenhang zwischen Roestelia koreænsis P. Henn. (Monsunia I, 1900, S. 5=Tremella koreænsis ARTH.) auf den Blättern von Pirus sinensis LindL. und Gymnosporangium japonicum durch Kulturversuche nach (Zeits. Pflanzenk., Bd. X, 1900, S. 1). Dieser Autor berichtet, dass die Sporenlager des Gymnosporangium nicht nur auf Stamm und $Z_{\text {weigen }}$ von Juniperus chinensis, wie die Original-Diagnose von Sydow sagt, sondern zuweilen auch auf den Blättern erscheinen. Die von ShIraI eruierte Tatsache ist in mehreren Arbeiten (ARThur (1900), Kleabahn (1904), Kern (1911), Sydow (1912), ClinToN (1913) u. m. a.) zitiert worden.

Doch haben K. Miyabe und G. Yamada, die seit langer Zeit die japanischen Gymnosporangien nebst anderen parasitischen Pilzen Japans zum Studium gemacht, die Rinden- und Nadelformen von Gymnosporangium auf Juniperus chinensis nicht als eine Art anzusehen vermocht. Um festzustellen, welche von diesen Ansichten richtig ist, müssen wir die Kulturversuche wiederholen, was sowohl für die Praktiker als auch für die Mykologen grosses Interesse darbietet.

Im Mai dieses Jahres ist es mir gegliickt, durch die Güte des Herrn G. Tanaka eine Sendung von kranken Juniperus-Stengeln (Rindenform) aus Hiratsuka (Prov. Sagami) zu erhalten. Davon übertrug ich eine Anzahl von aufgequollenen Sporenlagern auf die Blätter von Pirus sinensis Lindu. (am 10. u. 23. V), Pirus Malus L. (am 23. V), Amelanchier asiatica C. Koch. (am 23. V) und Pourthiza villosa DcNe. (=Photinia villosa DC.) (am 23. V), und bedeckte jeden inoculierten $Z_{\text {weig }}$ mit einem Papiersack, der nach 4 Tagen entfernt wurde. Nach 17 Tagen sah ich gelbe Flecke und 5 Wochen später Rostelia auf den Blättern von Pourthixa villosa; Birne, Apfel und Amelanchier blieben pilzfrei. Aus den Versuchen können wir ersehen, dass das stengelbewohnende Gymnosporangium von Juniperus chinensis nicht im genetischen Zusammenhang mit Rostelia koreænsis, sondern mit Ræestelia Photiniæ P. Henn. steht. (Hedw. XXX- 
III, 1894, S. 231=Gymnosp. Photiniz (P. Henn.) Kern, Bull. New York Bot. Gard., Vol. VII, No. 26, 1911, S. 443).

Nun bleibt die Frage ïber das nadelbewohnende Gymnosporangium noch offen. Nach der Beschreibung SHIRAIs befinden sich ,, die Sporenlager auf den Blättern und sind meist von konischer Form mit abgerundeter Spitze. Sie bilden isolierte Flecke, gewöhnlich einen oder zwei auf jedem Blatte." Meine Untersuchungen zeigen, dass in den morphologischen Eigenschaften diese Gymnosporangium-Species identisch ist mit Gymnosp. Haræanum Syd. (Ann. Mycol., Bd. X, 1912, S. 405; Monogr. Ured., III, 1912, S. 21). Es ist mir die Feststellung dieser Tatsache ermöglicht worden durch Herrn K. Haras freundliche Sendung von Originalspecimen-obgleich diese von der Originalbeschreibung Sydows, der ein altes und deformiertes Specımen vor sich hatte, mehr oder weniger abweichen. Leider konnte ich in diesem Jahre kein frisches Material des Pilzes zu Kulturversuchen erhalten.

Neuerdings hat K. HARA in dieser Zeitschrift (Vol. XXVII, No. 319, 1913, S. 348) mitgeteilt, dass er den Zusammenhang dieses Gymnosporangium mit Roestelia koreænsis auf Birnblättern durch Impfversuche (am 25. IV) feststellen konnte.

Vor mehreren Jahren stellten K. Mryabe and G. Yamada auch genaue Impfversuche an und fanden, dass die Keimschläuche der Teleutosporen des auf Blättern von Juniperus chinensis lebenden Gymnosporangium, das Gymnosp. asiaticum Miyabe (Bot. Mag. Tokyo, Vol. XVII, 1903, S. 34); Lehrbücher Yamadas und Inetas) in die Blätter von Pirus sinensis Lindr., Cydonia vulgaris Pers. und Cydonia japonica Pers. eindringen and Rostelia bilden. Es unterliegt keinem $Z_{\text {weifel, dass beide }}$ Species-Gymnosp. Haræanum und asiaticum-nach ihren morphologischen und biologischen Eigenschaften zu schliessen, zu einem und demselben Pilz gehören.

Wenn wir zum Schlusse nach Kerns Vorgang annehmen, dass alle Rœistelien zu Arten von Gymnosporangium gehören und jeder Artnamen nach der Priorität zu verändern ist, so müssen Gymnosp. japonicum als Gymnosp. Photiniz ; Gymnosp. Haræanum ( $=G$. asiaticum) als Gymnosp. koreænsis bezeichnet werden. 
Doch habe ich die Artnamen Gymnosp. japonicum und Gymnosp. Haræanum an dieser Stelle noch beibehalten.

\section{Napiculadium arundinacearum (CDA.) SACC.}

Bei uns in Hokkaido kommt an nassen Standorten die jährlich auf den fast abgestorbenen Blättern von Phragmites communis auftretende Napiculadium arundinacearum vor.

Die erste Mitteilung uiber das Vorkommen dieses Pilzes in Japan erfolgte 1909 von H. et P. Sydow, die das Material, das I. Miyake in der Provinz Izu (Atami) gesammelt hatte, in den Ann. Mycol., Bd. VII, S. 171 beschrieben haben.

In einer Arbeit „, Studies in Chinese Fungi “ in dieser Zeitschrift Vol. XXIV, No. 303, 1910, S. 63, hat I. MiYake einen neuen Pilz Brachysporium Phragmitis, welchen er auf den Blättern von Phragmites communis in Peking (Okt. 1910) gefunden hatte, beschrieben und auf P1. I, Fig. 10 w. 11 abgebildet. Obgleich ich das Original-Specimen noch nicht zu Gesicht bekommen habe, deuten Beschreibung und Abbildung zweifelsohne darauf hin, dass er Napiculadium arundinacearum vor sich gehabt hat. 